\title{
Cardiac autonomic modulation in drug-resistant epilepsy patients after vagus nerve stimulation therapy
}

\author{
Victor Constantinescu ${ }^{1}$, Daniela Matei ${ }^{1}$, Irina Constantinescu ${ }^{2}$, Dan Iulian Cuciureanu ${ }^{1}$ \\ ${ }^{1}$ University of Medicine and Pharmacy, Iasi, Romania \\ ${ }^{2}$ Department of Neurology, Regional Hospital of Orléans, Orléans, France
}

\begin{abstract}
The positive effect of vagus nerve stimulation (VNS) in patients with drug-resistant epilepsy is considered to be mediated by the afferent pathways of the vagus nerve, but the efferent pathways may influence the cardiac autonomic activity.

Aim of the study. To assess the effects of VNS on cardiac autonomic modulation in epilepsy patients, over three months of neurostimulation.

Clinical rationale for the study. Linear and non-linear heart rate variability (HRV) analysis can provide information on the sympathovagal balance and reveal particularities of the central control of the autonomic cardiovascular function.

Materials and Methods. Using Biopac Acquisition System, we analysed HRV parameters in resting condition and during sympathetic and parasympathetic activation tests in five patients with drug-resistant epilepsy, who underwent VNS procedure.

Results. During the sympathetic and vagal activation tests, all five patients presented normal responses of cardiac autonomic activity, reflected in RMSSD, HFnu and LF/HF dynamics in both HRV evaluations. No bradycardia, cardiac arrhythmia or orthostatic hypotension was registered during the two evaluations.

Conclusions. Our results indicate that VNS appears not to alter the cardiac autonomic function after three months of neurostimulation. HRV analysis is a useful tool for evaluating cardiac autonomic modulation in epilepsy patients during VNS therapy.

Clinical Implications. Patients with decreased HRV should be periodically monitored. Cardiac changes in patients with epilepsy are important because of the additional risk of arrhythmias mediated through the autonomic dysfunction.

Key words: drug-resistant epilepsy, vagus nerve stimulation, cardiac autonomic modulation, heart rate variability, linear and non-linear analysis
\end{abstract}

(Neurol Neurochir Pol 2020; 54 (4): 329-336)

\section{Introduction}

Since vagus nerve stimulation (VNS) was approved as a therapeutic approach for the treatment of refractory epilepsy, the search has been ongoing for nonpharmacological modulation of the autonomic nervous system (ANS) for different pathological conditions. The advantage of VNS therapy has also been evaluated for drug-resistant depression, heart failure, hypertension, and cardiac arrhythmias [1,2].

The precise mechanism of neuromodulation exerted by the VNS is still a matter of debate. $80 \%$ of the fibres of the vagus nerve are afferent pathways to the central nervous system.
Only 20\% are efferent pathways, some of them reaching the cardiovascular system [3]. The afferent pathways of the vagus nerve play an essential role in the neuromodulation process, influencing the interplay of various cortical networks probably involved in epileptogenic activity $[4,5]$. The activation of the vagal efferent pathways concerns the sinoatrial node and the cardiac conduction system [2,4]. Consequently, it may decrease the heart rate and reduce atrioventricular conduction and excitability of the His bundle.

Research dedicated to VNS's impact on cardiac rhythm has yielded contradictory results. A minor increase in sympathetic cardiovascular modulation without significant haemodynamic

Address for correspondence: Daniela Matei, University of Medicine and Pharmacy, Universitatii street No 16, 700115 lasi, Romania,

e-mail:dvm2202@yahoo.com 
effects, probably related to the activation of sympathetic pathways from the brainstem, has been reported [3]. Cardiac bradyarrhythmia is a rare complication during ongoing VNS therapy [6]. An increase in cardiac vagal modulation appears to play a cardioprotective role against sudden death [7].

Heart rate variability (HRV) describes the variations between consecutive heartbeats, known as RR intervals, on ECG recordings. Frequency-domain analysis allows the assessment of the global variation of a biologic signal, divided into its different spectral components [8]. The presence of different frequency spectra can be attributed to the modulation of the ANS on cardiovascular activity [9-11]. Time-domain indices of HRV evaluate the amount of variability in measurements of the interbeat interval. Mechanisms involved in cardiovascular regulation interact with each other in a non-linear manner $[12,13]$. Non-linear dynamics can be evaluated with the help of chaos theory, offering a more detailed perspective of the HRV. Fractal methods assess the scaling exponent of the signal which indicates the presence of fractal properties, or self-similarity of beat-to-beat intervals - the RR intervals on the ECG recordings [14]. Entropy measures have been widely used in HRV analysis, assessing the irregularity and complexity of HRV [15].

The rationale for implementing the analysis of the non-linear dynamics of HRV is thus to better understand the mechanisms of cardiac autonomic control. It has been demonstrated that alteration of fractal properties precedes the onset of fatal cardiac arrhythmias [16], as the increased regularity and the loss of complexity in the heart rate signal is related to the dysregulation of cardiac autonomic control $[17,18]$. A decreased HRV was initially shown to be predictive of mortality in the elderly population [19]. Moreover, in epilepsy patients, it appears to be associated with an increased risk of sudden unexpected death (SUDEP) [20]. Therefore, HRV analysis may identify patients with autonomic dysregulation at risk of fatal cardiac arrhythmias [20].

The same group of patients was previously assessed using Multiple Trigonometric Regressive Spectral analysis, using a different analytical approach [21]. The actual evaluation based on Fast Fourier Transform provides, in addition to the spectral power analysis, the non-linear appraisal of the HRV. Since this has not been previously described [21], we considered it worthy of further analysis.

\section{Clinical rationale for the study}

The clinical rationale of this study was to assess the effects of VNS on cardiovascular autonomic function in different physiological conditions in drug-resistant epilepsy patients, over three months of neurostimulation. The results of the study may be clinically useful for detecting a cardiac activity adjustment in the analysed epilepsy patients, and may contribute to better understanding of the effect of VNS on the autonomic cardiac activity.

\section{Methods}

ECG recordings of the first five patients with drug-resistant epilepsy who underwent VNS procedure, in our department, were analysed. In these patients, seizure control was not obtained within two years of multiple antiepileptic drug treatment. Epilepsy surgery was not a viable option in any of the five patients. Each patient had an ECG recording before VNS procedure and after three months of neurostimulation, during $\mathrm{ON}$ and OFF periods of the stimulation. All patients were monitored with prolonged EEG and ECG recordings before and after the autonomic tests (including night EEG), which excluded clinical or infraclinical seizures.

A standardised protocol consisting of a resting state ECG recording followed by four autonomic activation tests, each lasting for five minutes, was applied. Standardised conditions imposed the following criteria: ECG recording at the same time range after 30 minutes of clinostatism rest, in the absence of noise, at a constant temperature of $22^{\circ} \mathrm{C}$, without previous physical activity or the ingestion of beverages containing caffeine. The four autonomic activation tests were performed in the same sequence in all patients, as follows: deep breathing, standing, hand-grip and Valsalva manoeuvre. To remove the respiratory influence on the heart rate, the patients followed a paced breathing pattern at 15 cycles per minute, as described in other studies [22]. During the deep breathing test, a complete deep inhale and exhale lasted 10 seconds, six complete cycles per minute, to emphasise the vagal activation. The following sympathetic activation tests were performed: the standing test and the hand-grip test, consisting of a three-minute isometric contraction of the fist, using a dynamometer. BIOPAC ${ }^{\circ}$ acquisition system was used for the ECG recording. AcqKnowledge software version 3.9.1.6 eliminated artifacts of the recorded signal. HRV analysis was performed using Kubios HRV software version 2.2 (Biosignal Analysis and Medical Imaging Group, University of Eastern Finland). HRV parameters were analysed using Fast Fourier Transform. A minimum of 256 RR intervals on the ECG were analysed for each recording.

The following HRV time-domain parameters were analysed: Root Mean Square of the Successive Differences (RMSSD), the proportion of pairs of successive RR intervals that differ by more than $50 \mathrm{~ms}$ to the total number of NN intervals (pNN50), the standard deviation of the so-called normal-to-normal NN interval that reflects all the cyclic components responsible for variability in the period of recording (SDNN), the mean RR interval, and the heart rate [9]. The frequency-domain analysis referred to the following spectral components: VLF (very low frequency power, $0.02-0.04 \mathrm{~Hz}$ ), LF (low frequency power, 0.04-0.15 Hz) and HF (high frequency power, 0.15-0.4 Hz). These parameters were expressed in absolute values $\left(\mathrm{ms}^{2}\right)$, and in relative values calculated as a percentage (\%) to total power. Given the complex mechanisms that seem to influence the values of the VLF spectrum (i.e. thermoregulatory mechanisms, activity of the renin-angiotensin-aldosterone system) $[9$, 
10], we analysed the normalised units (nu) for the LF and HF spectrum, which excluded from calculation VLF values, and the LF/HF ratio (the ratio of absolute LF power to HF power). RMSSD, pNN50, HF and HFnu parameters are considered to be markers of parasympathetic autonomic control on the heart rate $[9,10]$.

The following HRV parameters were considered for the non-linear analysis: SD1, SD2, SampEn (Sample Entropy), ApEn (Approximate Entropy), DFA $\alpha 1$ and DFA $\alpha 2$ (Detrended Fluctuation Analysis a1 and a2). When analysing the Poincaré graph, SD1 shows the short-term variability of a chronological series, while SD2 is the second component of the ellipse formed from the point cloud (which has as abscissa and ordinate two consecutive R-R values from the ECG recording) and it illustrates the long-term variability of the biological signals [23]. SD1 represents the standard deviation of the Poincare plot and is graphically perpendicular to the line-of-identity, while SD2 is along the line-of-identity. SD1 is considered a parameter that reflects the influence of parasympathetic tone on the control of the sinus node, being an expression of the rapid changes of the RR interval, since the vagal effects on the sinus node manifest faster than those mediated by the sympathetic nervous system $[24,25]$. ApEn measures the 'disorder' in the heart rate signal and quantifies the regularity and complexity of the chronological series [26]. SampEn is a more constant measure derived from ApEn [27], which quantifies the complexity of the signal in short time segments [28], low values of SampEn indicating a greater similarity between successive RR in chronological series [29]. Detrended Fluctuation Analysis (DFA) is an evaluation method of the statistical 'self-affinity', assessing the regularity and complexity of the biosignals $[27,30,31]$ when the RR interval is analysed. Values of the scalar exponent $\alpha$ that are higher than 1 illustrate an increase in regularity and a decrease in signal complexity in the chronological series $[28,29]$, with an increased self-correlation power, constantly associated with pathological conditions $[32,33]$. al represents the short-range scaling exponent, while a2 represents the long-range scaling exponent [14].

We referred mainly to the SD1, SampEn, ApEn and DFA a1 parameters, as our ECG recordings lasted five minutes.

All five patients underwent left laterocervical stimulation of the vagus nerve and had no cardiovascular comorbidities or cardiovascular medication. The output current of stimulation was $2 \mathrm{~mA}$ for the first, third and fourth patients, $1.5 \mathrm{~mA}$ for the second patient, and $1 \mathrm{~mA}$ for the fifth patient. The frequency of stimulation was set at $30 \mathrm{~Hz}$ for all five patients, while pulse width $(500 \mu \mathrm{sec})$, duty cycle $(10 \%)$, ON time period (30 seconds) and OFF time period (5 minutes) was identical for all patients.

Biological parameters (blood pressure, oxygen saturation, renal and hepatic function and blood electrolytes) were within the normal range for all five patients. The antiepileptic medication was unchanged either in the three months before the first ECG recordings or between the two HRV tests. Blood pressure was measured in supine and orthostatic position for each patient after both evaluations.

Patients were recruited from the neurological department, and all patients were duly informed according to the study protocol and consented to the assessment in accordance with ethical principles. This study was carried out in accordance with the Helsinki Declaration.

For the statistical analysis of data, taking into consideration the small sample size, series normalisation was very difficult. Applied comparative tests were specific to the characteristics of the analysed parameters. A value of $\mathrm{p}<0.05$ was considered significant. GraphPad Prism software version 6.07 was used for the analysis and graphical presentation of the data.

\section{Description of patients}

The first patient, a 33-year-old female, was diagnosed with focal epilepsy (left anterior temporal epilepsy) and secondarily generalised seizures. The clinical symptoms were rotatory vertigo, breathing difficulties, facial flush, dreamy state, and generalisation. Brain MRI showed no abnormalities. The patient was being medicated with three antiepileptics: lamotrigine, levetiracetam and oxcarbazepine.

The second patient, a 34-year-old female, presented focal epilepsy (left insular epilepsy) with secondarily generalised seizures. The clinical symptoms were nausea, dyspnoea, abnormal sensation of retrosternal pain, burning heat restricted to the perioral area, and anarthria. No epilepsy-related brain MRI abnormalities were found. The patient was under treatment with valproic acid and levetiracetam.

The third patient, a 34-year-old female, had been diagnosed with focal epilepsy (right insular epilepsy) and secondarily generalised seizures (rotatory vertigo, facial flush, sense of unreality, and subsequent generalisation) at the age of 22 . No epilepsy-related brain MRI abnormalities. Patient under treatment with levetiracetam and oxcarbazepine.

The fourth patient, a 29-year-old female, presented multifocal epilepsy with secondarily generalised seizures (onset features: vertigo, sweating and motor unilateral symptoms, motor aphasia and generalisation). Brain MRI showed parietal and occipital gyration abnormalities. Pharmacological treatment consisted of lamotrigine, levetiracetam and carbamazepine.

The fifth patient, a 31-year-old male, presented left insular focal epilepsy with secondarily generalised seizures (retrosternal ascending heat, hypersalivation and post-ictal psychomotor agitation with hetero-aggressive behaviour). Left insular atrophy with frontoparietal extension was revealed on cerebral MRI. Antiepileptic medication consisted of valproate and oxcarbazepine.

\section{Results}

The first patient presented a sympathetic predominance on the heart rate control, as indicated by RMSSD, pNN50, HFnu and LF/HF values in resting state (Tab. 1). After the autonomic 
activation tests, the patient displayed an appropriate response of the cardiac autonomic regulation, as marked by the values of HFnu and LF/HF. Parasympathetic activation tests increased the HRV, illustrated by DFAal values (Tab. 1). There was no significant difference concerning the dynamic of HFnu and LF/ HF parameters in response to activation tests in the two HRV evaluations (Fig. 1). After three months of neurostimulation, an improvement in HRV was noticed, as shown by an increase of ApEn and SampEn during standing test, hand-grip test and Valsalva manoeuvre (Fig. 1).

The second patient presented normal responses to parasympathetic and sympathetic activation tests, reflected in HFnu, LF/HF and RMSSD values during challenge, in both HRV evaluations. A decrease in the HRV after sympathetic activation tests in both evaluations was seen regarding DFA al values (Fig.1).

The third patient presented appropriate responses to parasympathetic and sympathetic activation tests mirrored in the dynamics of HFnu and LF/HF ratio in both HRV evaluations. DFAa1 presented similar values after three months of neurostimulation (Fig.1).

The fourth patient presented normal dynamic of the HFnu and LF/HF ratio in response to autonomic activation tests, similar in both HRV assessments. During sympathetic activation tests, there was a shift to sympathetic predominance, seen in LF/HF values, correlated with a low HRV after three months of neurostimulation, revealed by DFAal values (Fig.1).

For the fifth patient, during the first evaluation, the Valsalva manoeuvre determined an increase of vagal modulation and of the HRV, illustrated by the values of RMSSD, pNN50, HFnu, SD1 and DFAa1 (Tab. 1). During the second evaluation, deep breathing test induced an increase in the parasympathetic control over the heart rate (higher RMSSD, HFnu values, lower $\mathrm{LF} / \mathrm{HF}$ ratio), compared to resting state, highlighting a normal response to the vagal activation test. ApEn and SampEn illustrated similar responses to the activation tests in both HRV evaluations (Fig. 1).

All five patients presented an increase of vagal modulation after parasympathetic activation tests, specifically at the Valsalva manoeuvre ( $\mathrm{p}<0.05)$, during the first HRV evaluation (T1) and after deep breathing test during the second HRV evaluation (T2), as shown by the RMSSD values (Fig. 2). LF/ HF ratio decreased after deep breathing test $(\mathrm{p}<0.05)$ and Valsalva manoeuvre during the first HRV evaluation. The same dynamic of the LF/HF values was observed during the second HRV evaluation, with increased values after standing and hand-grip tests $(\mathrm{p}<0.05)$ (Fig. 2). HFnu presented increased values after deep breathing test $(\mathrm{p}<0.05)$ during the first HRV evaluation. Similar features were observed at the second HRV evaluation, with an increment of the HFnu values after deep breathing test and a decrease after sympathetic activation tests $(\mathrm{p}<0.05)$, reflecting a regular response of the heart rate to sympathetic and parasympathetic modulation (Fig. 2). SDNN values presented a similar dynamic as RMSSD in response to the autonomic tests. Mean RR values were correlated to the heart rate values during the four autonomic tests (Tab. 1).

No patient presented orthostatic hypotension, defined as a decrease of at least $20 \mathrm{mmHg}$ in systolic blood pressure or of at least $10 \mathrm{mmHg}$ in diastolic blood pressure, after a three-minutes standing test, performed after ECG recording, incurred after the two HRV evaluations. During autonomic evaluations and the prolonged ECG recordings, no cardiac arrhythmias were identified for the five patients. Furthermore, the patients did not recall seizure-related symptoms during the current hospitalisation. During the autonomic tests performed, including the deep breathing test, the patients were not monitored by EEG, but no seizure was observed by the examiner or reported by the patient.

\section{Discussion}

The cortical neuromodulation exerted by VNS therapy involves brain structures related to autonomic regulation, such as the prefrontal region, thalamus and amygdala [34]. Recent findings indicate that activated vagal afferents initiate centrally mediated reflexes that inhibit parasympathetic efferent outflows to the heart [35], without consequent bradycardia, a clinical feature not found in our five patients after VNS therapy. Efferent vagal fibres do not directly synapse with cardiomyocytes, but rather with the intrinsic cardiac nervous system, acting as a buffer in modulating the commands to the cardiomyocytes [35]. The intrinsic cardiac nervous system comprises a complex network of ganglia and its neurons that can independently operate or connect with its complement structures of the autonomic pathways in the spinal cord, brainstem or cortex, in order to balance the intracardiac reflexes [35].

Periodic VNS may effectively modulate heart rate dynamics. A phenomenon of pharmacological tolerance has been described, in which both vagus nerve and the autonomic nervous network adapt to periodic stimulation [3].

Epilepsy and seizures can have dramatic effects on cardiac function, through the ANS. While some authors have concluded that VNS seems not to change decreased HRV in drug-resistant epilepsy patients [35], others have reported that VNS improves HRV shortly after implantation via the extensive innervation of the vagus nerve into the sinoatrial and atrioventricular nodes $[3,8,36]$. However, cardiac autonomic dysfunction related to VNS in epilepsy patients is rare (0.1\%) [37-39].

The originality of our study consists of using the autonomic activation tests (Ewing tests) and the analysis of non-linear HRV parameters besides time- and frequency-domain parameters for describing the cardiac autonomic response after sympathetic and parasympathetic challenge in patients with drug-resistant epilepsy, three months after vagal stimulation. Non-linear parameters have been used to analyse and predict the behaviour of biological phenomena. These parameters 
Table 1. Heart rate variability (HRV) parameters for the five patients

\begin{tabular}{|c|c|c|c|c|c|c|c|c|c|c|c|c|}
\hline $\begin{array}{l}\text { Test } 1 / \\
\text { /Test } 2\end{array}$ & HR & Mean RR & SDNN & RMSSD & pNN50 & LF (nu) & HF (nu) & LF/HF & SD1 & ApEn & SampEn & DFAa 1 \\
\hline RS Patient 1 & $90 / 91$ & $671 / 660$ & $39 / 31$ & $13 / 15$ & $0.6 / 0.5$ & $84.8 / 77.4$ & $14.7 / 22.5$ & $5.74 / 3.4$ & $16 / 10$ & $1.2 / 1.1$ & $1.3 / 1.3$ & $1.1 / 1.4$ \\
\hline DB Patient 1 & $92 / 87$ & $653 / 685$ & $48 / 36$ & $66 / 45$ & $1.7 / 0.5$ & $36.5 / 44.6$ & $56.4 / 55$ & $0.64 / 0.8$ & $46.8 / 32$ & $1.1 / 1$ & $1.1 / 1$ & $0.6 / 1.2$ \\
\hline ST Patient 1 & $92 / 96$ & $655 / 632$ & $62 / 25$ & $77 / 22$ & $2.1 / 2.8$ & $69 / 77$ & $30 / 22$ & $2.27 / 3.4$ & $54.5 / 15$ & $0.8 / 1.1$ & $0.8 / 1.1$ & $0.8 / 1.3$ \\
\hline HG Patient 1 & $92 / 87$ & $653 / 683$ & $72 / 22$ & $86 / 14$ & $2.7 / 0.3$ & $70 / 83.5$ & $29 / 16.5$ & $2.39 / 5$ & $60 / 10.4$ & $0.8 / 1.1$ & $0.7 / 1.6$ & $0.7 / 1.5$ \\
\hline VA Patient 1 & $92 / 93$ & $659 / 642$ & $69 / 35$ & $77 / 14$ & $2.6 / 1$ & $42.7 / 74.7$ & $57.3 / 25.2$ & $0.74 / 2.9$ & $50 / 10.4$ & $0.7 / 1$ & $0.7 / 1$ & $0.6 / 1.3$ \\
\hline RS Patient 2 & $76 / 67$ & $793 / 903$ & $49 / 67$ & $39 / 67$ & $12 / 36$ & $68 / 54.7$ & $31 / 45.3$ & $2.4 / 1.2$ & $28 / 47$ & $1.1 / 1$ & $1.6 / 1.6$ & $1.2 / 0.9$ \\
\hline DB Patient 2 & $78 / 73$ & $777 / 824$ & $72 / 54$ & $89 / 53$ & $9.2 / 14.5$ & $38.7 / 52.9$ & $60.9 / 47$ & $0.63 / 1.1$ & $63 / 38$ & $1 / 1$ & $1 / 1.3$ & $0.9 / 1.1$ \\
\hline ST Patient 2 & $85 / 75$ & $711 / 802$ & $80 / 58$ & $58 / 42$ & $4.5 / 14.5$ & $71 / 77$ & $28 / 22$ & $2.53 / 3.3$ & $41 / 29$ & $0.7 / 1$ & $0.6 / 1.3$ & $1.1 / 1.2$ \\
\hline HG Patient 2 & $76 / 76$ & 787/797 & $40 / 58$ & $40 / 44$ & $12.3 / 15.8$ & $58 / 78.4$ & $41.7 / 21.5$ & $1.39 / 3.6$ & $29 / 31$ & $1.2 / 1.1$ & $1.6 / 1.4$ & $1.1 / 1.3$ \\
\hline VA Patient 2 & $81 / 70$ & $740 / 863$ & $75 / 57$ & $96 / 51$ & $6.3 / 20$ & $41 / 71$ & $58.7 / 28.6$ & $0.69 / 2.4$ & $68 / 36$ & $0.8 / 1$ & $0.8 / 1.4$ & $0.6 / 1.1$ \\
\hline RS Patient 3 & $89 / 81$ & $670 / 735$ & $41 / 24$ & $20 / 20$ & $2.4 / 1.7$ & $73.9 / 70$ & $26 / 29.5$ & $2.84 / 2.3$ & $14 / 14$ & $1 / 1.2$ & $1.1 / 1.7$ & $1.3 / 1.2$ \\
\hline DB Patient 3 & $90 / 81$ & $665 / 740$ & $30 / 29$ & $20 / 23$ & $3.6 / 2.7$ & $50 / 63.9$ & $50 / 36$ & $0.99 / 1.7$ & $14 / 16$ & $1 / 1.1$ & $1.1 / 1.6$ & $1.3 / 1.2$ \\
\hline ST Patient 3 & $93 / 86$ & $640 / 700$ & $19 / 29$ & $14 / 22$ & $0.9 / 2.8$ & $73 / 78$ & $26 / 20$ & $2.77 / 3.8$ & $10 / 16$ & $1.2 / 1.1$ & $1.7 / 1.6$ & 1.3/1.2 \\
\hline HG Patient 3 & $89 / 83$ & $673 / 724$ & $22 / 32$ & $18 / 24$ & $0.2 / 4.4$ & $80 / 81.8$ & $20 / 18.2$ & $3.99 / 4.4$ & $12.7 / 17$ & $1.2 / 1$ & $1.7 / 1.4$ & $1.2 / 1.4$ \\
\hline VA Patient 3 & $89 / 78$ & $672 / 766$ & $39 / 36$ & $28 / 29$ & $4.4 / 7$ & $78 / 63$ & $21 / 36$ & $3.6 / 1.72$ & $20 / 21$ & $1 / 1$ & $1.2 / 1.6$ & 1.4/1.2 \\
\hline RS Patient 4 & $79 / 74$ & $755 / 811$ & $9 / 16$ & $11 / 13.9$ & $0.4 / 0.3$ & $77 / 76$ & $22 / 23$ & $3.5 / 3.2$ & $35 / 9$ & $0.8 / 1.1$ & $0.8 / 1.6$ & $1 / 1.4$ \\
\hline DB Patient 4 & $76 / 72$ & $790 / 823$ & $31 / 18$ & $28 / 15$ & $4 / 1.2$ & $73 / 52$ & $26.9 / 47$ & 2.7/1.1 & $20 / 11$ & $1 / 1.1$ & $1.1 / 1.6$ & $1.2 / 1.1$ \\
\hline ST Patient 4 & $82 / 81$ & $737 / 743$ & $27 / 25$ & $19 / 17$ & $2.5 / 0.9$ & $84 / 81$ & $15 / 18$ & $5.5 / 4.4$ & $14 / 12$ & $0.9 / 0.9$ & $1.2 / 0.6$ & $1.4 / 1.2$ \\
\hline HG Patient 4 & $81 / 76$ & $743 / 787$ & $40 / 17$ & $37 / 13$ & $2.5 / 0.5$ & $73.7 / 81$ & $26.1 / 18$ & $2.82 / 4.3$ & $26 / 9$ & $0.9 / 1.2$ & $0.9 / 1.5$ & $0.5 / 1.3$ \\
\hline VA Patient 4 & $76 / 72$ & $792 / 826$ & $46 / 32$ & $58 / 23$ & $2.3 / 4.9$ & $60.4 / 71$ & $39.3 / 28$ & $1.52 / 2.5$ & $41 / 16$ & $0.9 / 1$ & $0.9 / 1.3$ & $0.9 / 1.2$ \\
\hline RS Patient 5 & $75 / 73$ & $805 / 814$ & $85 / 43$ & $54 / 29.9$ & $25 / 6.9$ & $62 / 58.3$ & $37 / 41.7$ & $1.68 / 1.3$ & $38.8 / 21$ & $1 / 1.2$ & $1.2 / 1.6$ & $1.1 / 1$ \\
\hline DB Patient 5 & $74 / 76$ & $811 / 791$ & $87 / 88$ & $52 / 111.5$ & $27 / 6.8$ & $50.9 / 43.7$ & $48.9 / 55$ & $1.04 / 0.7$ & $36.8 / 78$ & $1 / 0.8$ & $1.1 / 0.7$ & $1.2 / 0.9$ \\
\hline ST Patient 5 & $81 / 77$ & $754 / 782$ & $104 / 93$ & $59.8 / 24.6$ & $20.1 / 5.1$ & $65.2 / 65.2$ & $34.6 / 34.8$ & $1.88 / 1.8$ & $42.3 / 17$ & $0.8 / 0.7$ & $0.8 / 0.5$ & $1.1 / 1.4$ \\
\hline HG Patient 5 & $75 / 77$ & $805 / 776$ & $93 / 42$ & $57.8 / 20.2$ & $27.8 / 2.5$ & $66.1 / 73.4$ & $33.8 / 26.5$ & $1.95 / 2.7$ & $40.9 / 14$ & $1 / 1$ & $1.1 / 1.2$ & $1.1 / 1.2$ \\
\hline VA Patient 5 & $69 / 75$ & $883 / 795$ & $132 / 46$ & $146 / 24.2$ & $52.4 / 4.7$ & $39.2 / 84.9$ & $60.2 / 15.1$ & $0.65 / 5.6$ & $104 / 17$ & $1.1 / 1$ & $1.4 / 1.2$ & $0.8 / 1.4$ \\
\hline
\end{tabular}

$\mathrm{HR}$ - heart rate; RR interval — variations between consecutive heartbeats; SDNN — standard deviation of Normal-to-Normal intervals; RMSSD — Root Mean Square of Successive Differences; pNN50 - the proportion of NN50, representing the number of pairs of successive NNs that differ by more than $50 \mathrm{~ms}$, divided by total number of NNs; LF - low frequency power; $\mathrm{HF}-$ high frequency power; ApEn - Approximate Entropy; SampEn - Sample Entropy; SD1 - standard deviation of instantaneous beat-to-beat interval variability; DFA - detrended fluctuation analysis

have proved to be good predictors of morbidity and mortality in clinics [40].

Although it is known that drug-resistant epilepsy is associated with significant inhibition of vagal modulation of heart rate and lower HRV $[41,42]$, the non-linear parameters ApEn, SampEn, SD1 underlined an increase of HRV during the vagal activation tests compared to sympathetic activation tests. DFA a 1 confirmed the increase of HRV, especially during the Valsalva test, in all five patients, in the first evaluation. Thus, the non-linear analysis of HRV validated the results from time- and frequency-domain analysis, reflecting the shift in the sympathovagal balance during the autonomic tests.

VNS appears not to disrupt the cardiac autonomic activity, with no significant alteration in HRV parameters during autonomic tests being registered during the ECG recordings. The first and fourth patients presented sympathetic predominance over the heart rate control. The first patient displayed an increase of HRV, while the fourth patient displayed a decrease of HRV requiring further cardiac monitoring. Also, the second patient presented a decrease of HRV after sympathetic activation tests in both evaluations, while the third and the fifth patients kept constant features regarding the non-linear parameters. This observation underlines the importance of non-linear analysis, which may provide useful information about the cardiac autonomic state.

Our results reveal that all patients presented adequate autonomic responses after sympathetic and parasympathetic activation tests, before VNS therapy, and three months after neurostimulation.

Although not necessarily reflecting a novel mechanism, the dynamic autonomic tests provide useful information regarding cardiac regulation. An impaired response to the autonomic challenge reflected by HRV parameters is not only a potential biomarker for monitoring progressive decline of the ANS system regulation, but is also a probable risk factor for sudden unexplained death determined by cardiac arrhythmias in patients with epilepsy [20,43].

Further studies are needed to assess whether a perpetuation of the sympathetic control and a decreased HRV, observed in some of our patients during sympathetic activation 


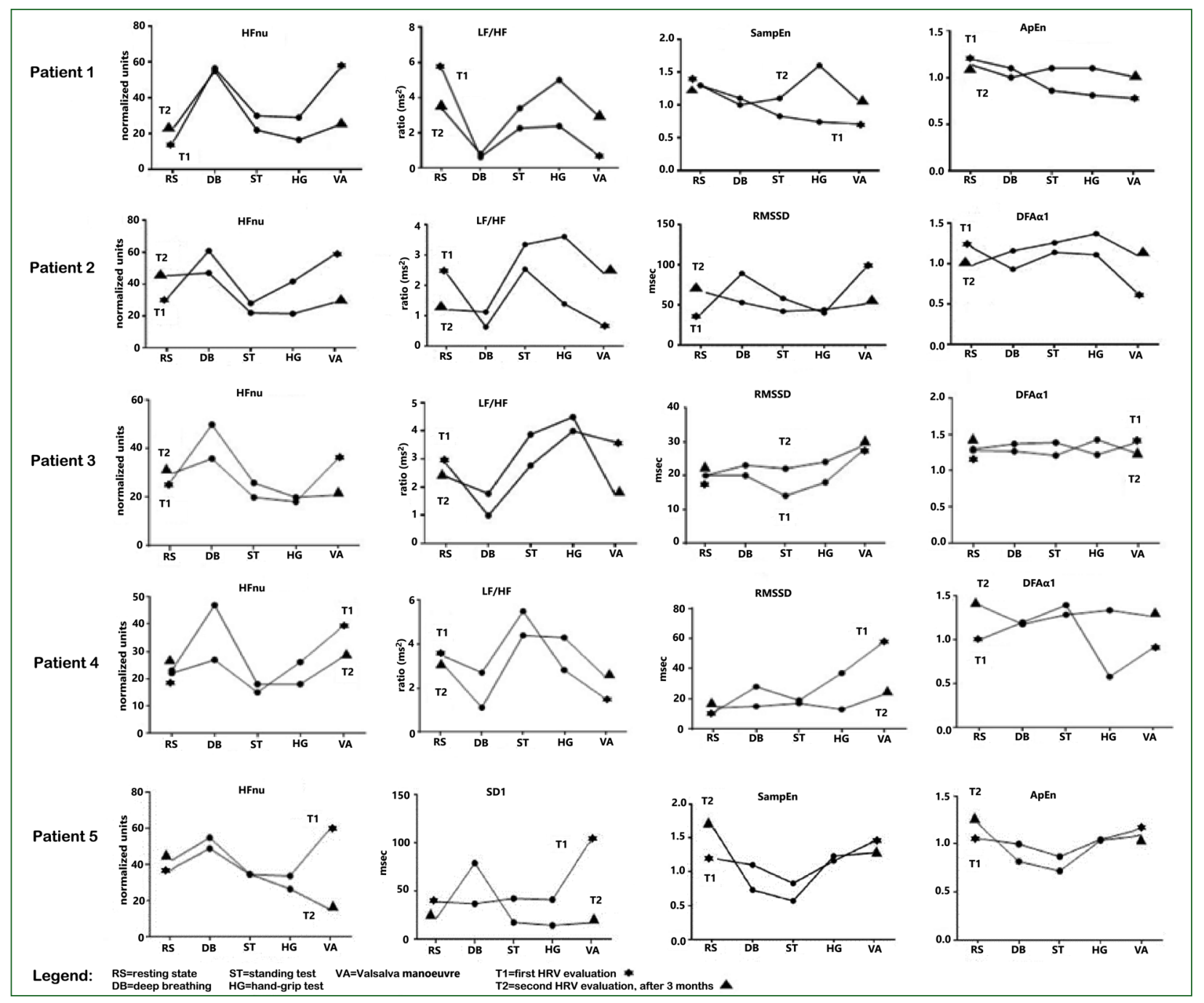

Figure 1. Heart rate variability (HRV) parameters for the five patients

tests, is confirmed in larger populations of drug-resistant epilepsy patients during VNS therapy, and if it might predispose to negative outcomes in patients with concurrent diseases. Currently, there is insufficient data to show how VNS influences different cardiac autonomic activity parameters within 24 hours, implying sleep-wake alternation, including physiological vulnerability periods of cardio-circulatory or respiratory control.

HRV analysis may be included in the current drug-resistant epilepsy patient evaluation. Drug-resistant epilepsy patients who are non-responders to VNS therapy, defined as the lack of an at least $50 \%$ seizure reduction after one year of treatment, had significantly lower RMSSD, pNN50, HF, and SD1 than the responders [44]. Thus, presurgical HRV evaluation measurements representing parasympathetic control on heart rate were significantly associated with the responsiveness to VNS [44] and may serve as a marker for the effectiveness of this therapeutic option, although further studies are needed to evaluate this hypothesis.
VNS has been shown to exert antiarrhythmic effects, improve left ventricular function, and reduce mortality in patients with heart failure [45]. The optimum VNS parameters define a stabilised state in which both afferent and efferent fibres are activated in a balanced manner, called 'neural fulcrum' [35]. VNS performed near this neural fulcrum ensures an adequate response to stressors involving both central and peripheral components [35]. It would be of interest to analyse whether reaching this cardiac autonomic balanced state would reduce the risk of fatal cardiac arrhythmia in epilepsy patients.

HRV study could, therefore, provide essential data about the neural fulcrum and could guide the adjustments of VNS parameters for the neurological target as well.

One limit of our study, besides the limited number of patients, is the interfering antiepileptic medication, especially sodium or potassium channel blockers that may alter the depolarisation-repolarisation potentials of the cardiac cells. It is therefore difficult to distinguish the medication-mediated 


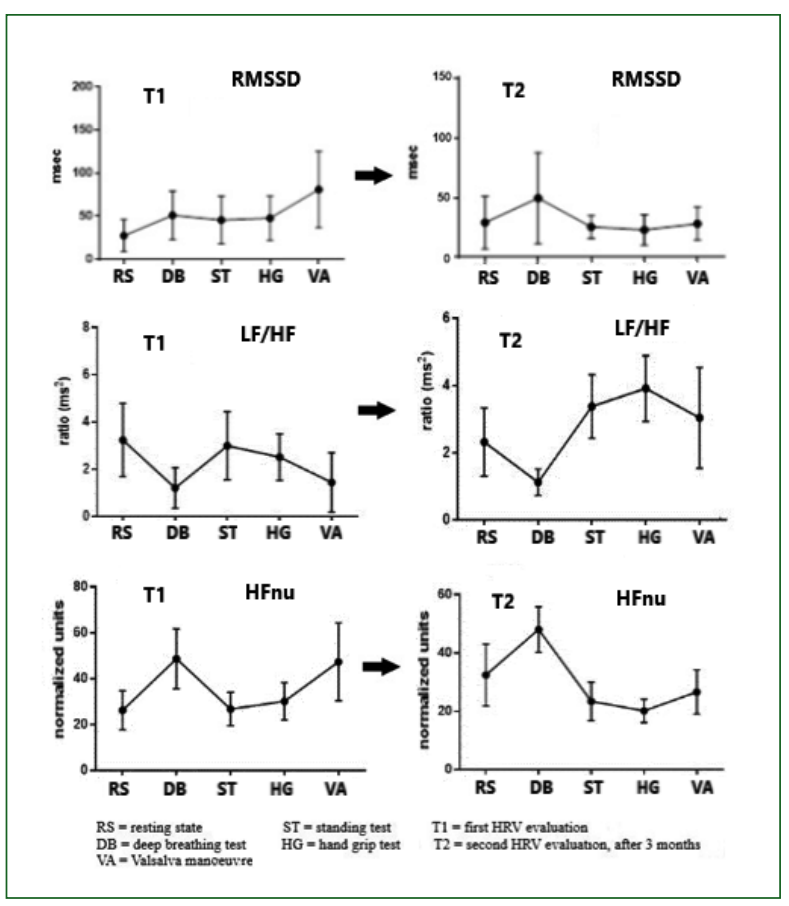

Figure 2. Root Mean Square of the Successive Differences (RM$\mathrm{SSD}$ ), low frequency power (LF)/ high frequency power (HF) ratio and HFnu dynamics for the five patients

effects from the cardiac dysautonomia present in epilepsy patients.

\section{Clinical implications and future directions}

The involvement of ANS in patients with epilepsy has been insufficiently explored, and has produced conflicting results. Epilepsy patients present a risk of sudden unexpected death, autonomic dysfunction being one of the causes. However, the exact mechanism remains unclear. HRV is a useful method to assess the influence of ANS at the cardiac level.

Our results revealed that VNS does not alter the cardiac autonomic responses to the sympathetic and parasympathetic activation tests, having no clinically relevant effects on cardiac autonomic activity at the analysed stimulation threshold.

Patients with decreased HRV should be periodically monitored. Further studies on larger groups of drug-resistant epilepsy patients, and longer follow-up periods, are needed in order to observe the cardiac autonomic response after neurostimulation.

\section{Conflicts of interest}

None.

\section{References}

1. Lee SW, Kulkarni K, Annoni EM, et al. Stochastic vagus nerve stimulation affects acute heart rate dynamics in rats. PLoS One. 2018;
13(3): e0194910, doi: 10.1371/journal.pone.0194910, indexed in Pubmed: 29590213.

2. Premchand RK, Sharma K, Mittal S, et al. Extended Follow-Up of Patients With Heart Failure Receiving Autonomic Regulation Therapy in the ANTHEM-HF Study. J Card Fail. 2016; 22(8): 639-642, doi: 10.1016/j.cardfail.2015.11.002, indexed in Pubmed: 26576716.

3. Garamendi I, Acera M, Agundez M, et al. Cardiovascular autonomic and hemodynamic responses to vagus nerve stimulation in drug-resistant epilepsy. Seizure. 2017; 45: 56-60, doi: 10.1016/j.seizure.2016.11.018, indexed in Pubmed: 27919011.

4. Henry TR. Therapeutic mechanisms of vagus nerve stimulation. Neurology. 2002; 59: S3-14.

5. Schachter SC, Saper CB. Vagus nerve stimulation. Epilepsia. 1998; 39(7): 677-686, doi: 10.1111/j.1528-1157.1998.tb01151.x, indexed in Pubmed: 9670894.

6. Amark P, Stödberg T, Wallstedt L. Late onset bradyarrhythmia during vagus nerve stimulation. Epilepsia. 2007; 48(5): 1023-1024, doi: 10.1111/j.1528-1167.2007.01023.x, indexed in Pubmed: 17381444.

7. Schomer AC, Nearing BD, Schachter SC, et al. Vagus nerve stimulation reduces cardiac electrical instability assessed by quantitative T-wave alternans analysis in patients with drug-resistant focal epilepsy. Epilepsia. 2014; 55(12): 1996-2002, doi: 10.1111/epi.12855, indexed in Pubmed: 25470430.

8. Bernardi L, Bianchini B, Spadacini G, et al. Demonstrable cardiac reinnervation after human heart transplantation by carotid baroreflex modulation of RR interval. Circulation. 1995; 92(10): 2895-2903, doi: 10.1161/01.cir.92.10.2895, indexed in Pubmed: 7586257.

9. Heart rate variability: standards of measurement, physiological interpretation and clinical use. Task Force of the European Society of Cardiology and the North American Society of Pacing and Electrophysiology. Circulation. 1996; 93(5): 1043-1065, indexed in Pubmed: 8598068.

10. Hilz MJ. Quantitative autonomic functional testing in clinical trials. In: Brown WF, Bolton CF, Aminoff MJ. ed. Neuromuscular function and disease. Basic, clinical and electrodiagnostic aspects. WB Saunders, Philadelphia 2002: 1899-1929.

11. Saul JP, Berger RD, Albrecht $P$, et al. Transfer function analysis of the circulation: unique insights into cardiovascular regulation. Am J Physiol. 1991; 261(4 Pt 2): H1231-H1245, doi: 10.1152/ ajpheart.1991.261.4.H1231, indexed in Pubmed: 1928405.

12. Huikuri HV, Mäkikallio TH, Perkiömäki J. Measurement of heart rate variability by methods based on nonlinear dynamics. J Electrocardiol. 2003; 36 Suppl: 95-99, doi: 10.1016/j.jelectrocard.2003.09.021, indexed in Pubmed: 14716599.

13. Voss A, Kurths J, Kleiner HJ, et al. The application of methods of non-linear dynamics for the improved and predictive recognition of patients threatened by sudden cardiac death. Cardiovasc Res. 1996; 31(3): 419-433, indexed in Pubmed: 8681329.

14. Golińska AK. Detrended fluctuation analysis (DFA) in biomedical signal processing: Selected examples. Studies in Logic, Grammar and Rhetoric. 2012; 29: 107-115.

15. Chen C, Jin Yu, Lo IL, et al. Complexity Change in Cardiovascular Disease. Int J Biol Sci. 2017; 13(10): 1320-1328, doi: 10.7150/ ijbs.19462, indexed in Pubmed: 29104498.

16. Mäkikallio $T H$, Koistinen J, Jordaens $L$, et al. Heart rate dynamics before spontaneous onset of ventricular fibrillation in patients with healed myocardial infarcts. Am J Cardiol. 1999; 83(6): 880-884, doi: 10.1016/s0002-9149(98)01068-6, indexed in Pubmed: 10190403.

17. Mäkikallio TH, Seppänen T, Niemelä $M$, et al. Abnormalities in beat to beat complexity of heart rate dynamics in patients with a previous 
myocardial infarction. J Am Coll Cardiol. 1996; 28(4): 1005-1011, doi: 10.1016/s0735-1097(96)00243-4, indexed in Pubmed: 8837582.

18. Varadhan R, Chaves PHM, Lipsitz LA, et al. Frailty and impaired cardiac autonomic control: new insights from principal components aggregation of traditional heart rate variability indices. J Gerontol A Biol Sci Med Sci. 2009; 64(6): 682-687, doi: 10.1093/gerona/glp013, indexed in Pubmed: 19223607.

19. Tsuji H, Venditti FJ, Manders ES, et al. Reduced heart rate variability and mortality risk in an elderly cohort. The Framingham Heart Study. Circulation. 1994; 90(2): 878-883, doi: 10.1161/01.cir.90.2.878, indexed in Pubmed: 8044959.

20. Myers KA, Bello-Espinosa LE, Symonds JD, et al. Heart rate variability in epilepsy: A potential biomarker of sudden unexpected death in epilepsy risk. Epilepsia. 2018; 59(7): 1372-1380, doi: 10.1111/ epi.14438, indexed in Pubmed: 29873813.

21. Constantinescu V, Matei D, Constantinescu I, et al. Heart Rate Variability and Vagus Nerve Stimulation in Epilepsy Patients. Transl Neurosci. 2019; 10: 223-232, doi: 10.1515/tnsci-2019-0036, indexed in Pubmed: 31497318.

22. Budrejko S, Kempa M, Chmielecka M, et al. Analysis of Heart Rate Variability During Head-Up Tilt-Test in Patients with Vasovagal Syncope. Eur J Transl Clin Med. 2018; 1(1): 24-36, doi: 10.31373/ ejtcm/92837.

23. Shaffer F, Ginsberg JP. An Overview of Heart Rate Variability Metrics and Norms. Front Public Health. 2017; 5: 258, doi: 10.3389/ fpubh.2017.00258, indexed in Pubmed: 29034226.

24. Mourot L, Bouhaddi M, Perrey S, et al. Decrease in heart rate variability with overtraining: assessment by the Poincaré plot analysis. Clin Physiol Funct Imaging. 2004; 24(1): 10-18, doi: 10.1046/j.1475-0961.2003.00523.x, indexed in Pubmed: 14717743.

25. Mourot L, Bouhaddi M, Perrey S, et al. Quantitative Poincaré plot analysis of heart rate variability: effect of endurance training. Eur J Appl Physiol. 2004; 91(1): 79-87, doi: 10.1007/s00421-003-0917-0, indexed in Pubmed: 12955518.

26. Peng CK, Havlin S, Stanley HE, et al. Quantification of scaling exponents and crossover phenomena in nonstationary heartbeat time series. Chaos. 1995; 5(1): 82-87, doi: 10.1063/1.166141, indexed in Pubmed: 11538314.

27. Corino VDA, Ziglio F, Lombardi F, et al. Detrended Fluctuation Analysis of Atrial Signal during Adrenergic Activation in Atrial Fibrillation. Computers in Cardiology. 2006; 33: 141-144.

28. Yeh RG, Shieh JS, Chen GY, et al. Detrended fluctuation analysis of short-term heart rate variability in late pregnant women. Auton Neurosci. 2009; 150(1-2): 122-126, doi: 10.1016/j.autneu.2009.05.241, indexed in Pubmed: 19464962.

29. Rodriguez E, Echeverria J, Alvarez-Ramirez J. Detrended fluctuation analysis of heart intrabeat dynamics. Physica A. 2007; 384(2): 429438, doi: 10.1016/j.physa.2007.05.022.

30. Acharya RU, Lim CM, Joseph P. Heart rate variability analysis using correlation dimension and detrended fluctuation analysis. ITBM-RBM. 2002; 23(6): 333-339, doi: 10.1016/s1297-9562(02)90002-1.

31. Absil PA, Sepulchre R, Bilge A, et al. Nonlinear analysis of cardiac rhythm fluctuations using DFA method. Physica A: Statistical Mechanics and its Applications. 1999; 272(1-2): 235-244, doi: 10.1016/ s0378-4371(99)00295-2.

32. Germán-Salló Z, Germán-Salló M. Non-linear Methods in HRV Analysis. Procedia Technology. 2016; 22: 645-651, doi: 10.1016/j.protcy.2016.01.134.
33. Pikkujämsä SM, Mäkikallio TH, Sourander LB, et al. Cardiac interbeat interval dynamics from childhood to senescence : comparison of conventional and new measures based on fractals and chaos theory. Circulation. 1999; 100(4): 393-399, doi: 10.1161/01.cir.100.4.393, indexed in Pubmed: 10421600.

34. Fornai F, Ruffoli R, Giorgi FS, et al. The role of locus coeruleus in the antiepileptic activity induced by vagus nerve stimulation. Eur J Neurosci. 2011; 33(12): 2169-2178, doi: 10.1111/j.1460-9568.2011.07707.x, indexed in Pubmed: 21535457.

35. Ardell JL, Rajendran PS, Nier HA, et al. Central-peripheral neural network interactions evoked by vagus nerve stimulation: functional consequences on control of cardiac function. Am J Physiol Heart Circ Physiol. 2015; 309(10): H1740-H1752, doi: 10.1152/ajpheart.00557.2015, indexed in Pubmed: 26371171.

36. Galbarriatu L, Pomposo I, Aurrecoechea J, et al. Vagus nerve stimulation therapy for treatment-resistant epilepsy: a 15-year experience at a single institution. Clin Neurol Neurosurg. 2015; 137: 89-93, doi: 10.1016/j.clineuro.2015.06.023, indexed in Pubmed: 26164349.

37. Ryvlin P, Gilliam FG, Nguyen DK, et al. The long-term effect of vagus nerve stimulation on quality of life in patients with pharmacoresistant focal epilepsy: the PuLsE (Open Prospective Randomized Long-term Effectiveness) trial. Epilepsia. 2014; 55(6): 893-900, doi: 10.1111/ epi.12611, indexed in Pubmed: 24754318.

38. Stemper B, Devinsky 0, Haendl T, et al. Effects of vagus nerve stimulation on cardiovascular regulation in patients with epilepsy. Acta Neurol Scand. 2008; 117(4): 231-236, doi: 10.1111/j.1600-0404.2007.00944.x, indexed in Pubmed: 18005223.

39. Ben-Menachem E. Vagus nerve stimulation, side effects, and long-term safety. J Clin Neurophysiol. 2001; 18(5): 415-418, doi: 10.1097/00004691-200109000-00005, indexed in Pubmed: 11709646

40. Vanderlei LC, Pastre CM, Hoshi RA, et al. Basic notions of heart rate variability and its clinical applicability. Rev Bras Cir Cardiovasc. 2009; 24(2): 205-217, doi: 10.1590/s0102-76382009000200018, indexed in Pubmed: 19768301.

41. Ronkainen E, Korpelainen JT, Heikkinen E, et al. Cardiac autonomic control in patients with refractory epilepsy before and during vagus nerve stimulation treatment: a one-year follow-up study. Epilepsia. 2006; 47(3): 556-562, doi: 10.1111/j.1528-1167.2006.00467.x, indexed in Pubmed: 16529621.

42. Liu H, Yang Z, Meng F, et al. Deceleration and acceleration capacities of heart rate in patients with drug-resistant epilepsy. Clin Auton Res. 2019; 29(2): 195-204, doi: 10.1007/s10286-018-0569-0, indexed in Pubmed: 30328033.

43. Baysal-Kirac L, Serbest NG, Şahin E, et al. Analysis of heart rate variability and risk factors for SUDEP in patients with drug-resistant epilepsy. Epilepsy Behav. 2017; 71(Pt A): 60-64, doi: 10.1016/j. yebeh.2017.04.018, indexed in Pubmed: 28549245.

44. Liu H, Yang Z, Huang L, et al. Heart-rate variability indices as predictors of the response to vagus nerve stimulation in patients with drug-resistant epilepsy. Epilepsia. 2017; 58(6): 1015-1022, doi: 10.1111/epi.13738, indexed in Pubmed: 28440954.

45. Rousselet L, Le Rolle V, Ojeda D, et al. Influence of Vagus Nerve Stimulation parameters on chronotropism and inotropism in heart failure. Conf Proc IEEE Eng Med Biol Soc. 2014; 2014: 526-529, doi: 10.1109/EMBC.2014.6943644, indexed in Pubmed: 25570012. 